\title{
How to use MATLAB to fit the ex-Gaussian and other probability functions to a distribution of response times
}

\author{
Yves Lacouture \\ Université Laval \\ Denis Cousineau \\ Université de Montréal
}

\begin{abstract}
This article discusses how to characterize response time (RT) frequency distributions in terms of probability functions and how to implement the necessary analysis tools using MATLAB. The first part of the paper discusses the general principles of maximum likelihood estimation. A detailed implementation that allows fitting the popular ex-Gaussian function is then presented followed by the results of a Monte Carlo study that shows the validity of the proposed approach. Although the main focus is the ex-Gaussian function, the general procedure described here can be used to estimate best fitting parameters of various probability functions. The proposed computational tools, written in MATLAB source code, are available through the Internet.
\end{abstract}

In recent years there has been an upsurge of interest in the response times (RT) of cognitive processes. This can be attributed in part to the wide availability of computer programs that allow experiments to be conducted automatically and RT to be measured with millisecond precision. However, and perhaps more importantly, many researchers see RT as major constraints when testing models of cognitive processes. Although RT are now routinely measured and frequently reported, there is a lack of standard tools for characterizing RT distributions. The difficulty is that simple descriptive statistics usually do not provide an adequate characterization of the data, and a

Address correspondence to: Yves Lacouture, École de Psychologie, Université Laval, Quebec City, Quebec, Canada G1K 7P4. Email: yves.lacouture@psy.ulaval.ca

This work was supported by research and equipment grants from the Natural Sciences and Engineering Research Council of Canada (NSERC) to both authors. We would like to thank Gene Bourgeau for his assistance in revising the article. description in terms of probabilistic functions is generally more suitable.

For instance, Figure 1 presents three data sets having the same mean $\bar{x}=300$. Each panel on Figure 1 shows the frequency distribution of 250 response times sampled from a specific population, each one characterized by a specific distribution. Although the mean of all three distributions is the same, the shapes are different. Panel (A) shows the distribution of data sampled from a population for which the distribution of response times has the shape of an exponential function. Panel (B) presents the resulting distribution of response times sampled from a process for which the data follow a normal (Gaussian) distribution. Finally, Panel (C) shows the resulting distribution of response times obtained when the underlying process yield data following the ex-Gaussian function (the usefulness of this function for characterizing a distribution will be discussed later). A distribution of response time that matches the shape of an exponential function is believed to be characteristic of decision processes (see Luce, 1986, chap. 

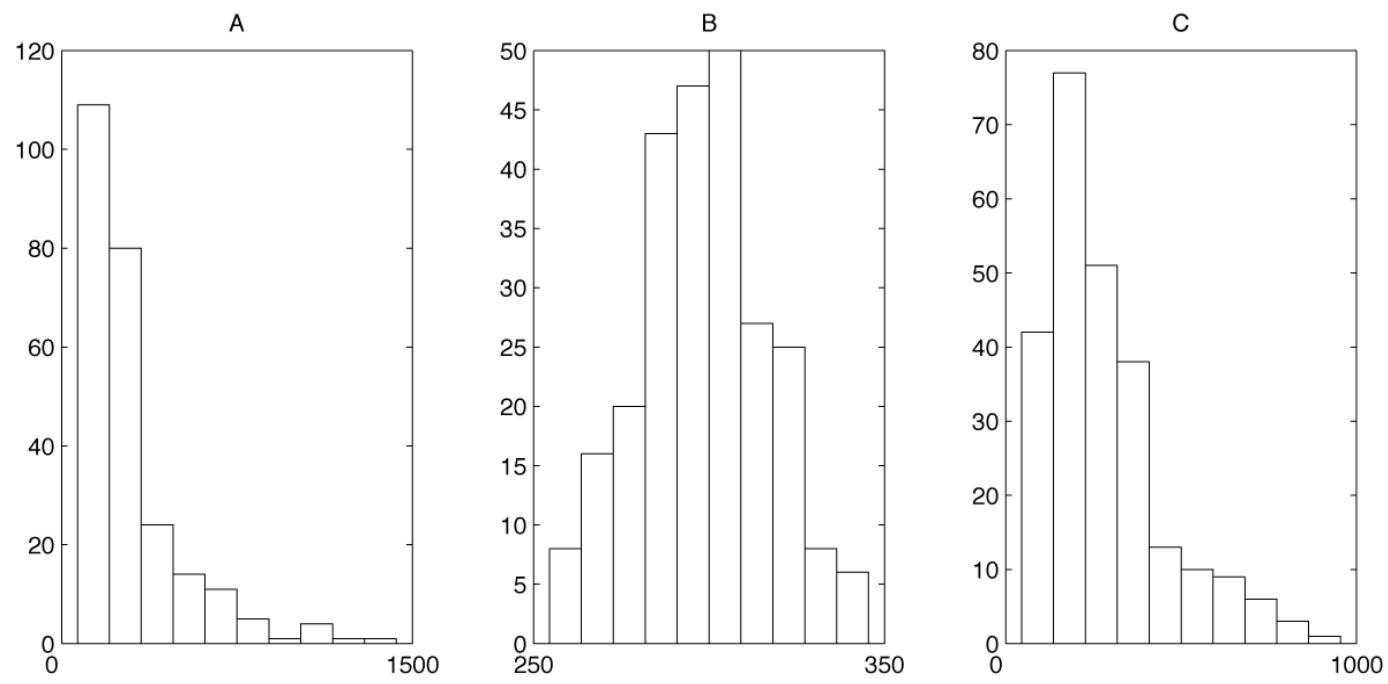

Figure 1. Three distributions of response times sampled from different populations each characterized by a specific probability functions. Panel (A) shows a distribution of response times shaped like an exponential function. Panel (B) shows data following a normal distribution and Panel (C) presents a distribution of data matching the ex-Gaussian function. All three distributions have the same mean $\bar{x}=300$.

6). A normal distributions of response times is typical of a large variety of human performances including perceptual and motor processes. Finally, distributions of response times that follow the ex-Gaussian function results of two or more sequential processes made of a mixture of exponential and normal distributions. Characterizing the shape of a distribution of responses times allows a better description of the results but also permits testing hypothesis about the underlying cognitive processes.

Finding the best matching distribution to a set of response times provides information about the underlying processes, but also the specific parameter values of the best fitting function allows to test specific hypothesis. There are a large number of probabilistic functions that can be used to characterize a set of response times. The use of some functions is theoretically justified. Other functions yield parameter values easier to interpret. Among the wide variety of functions that can be fitted to characterized a distribution of response times, the ex-Gaussian function is especially popular because it is theoretically justified and also because it provides parameter values that are easy to interpret. For that reason, the ex-Gaussian is the main focus of this paper.

Several researchers have shown that distribution analysis allows finding results that are not revealed by usual statistics. Among them, Heahtcote, Popiel \& Mewhort (1991) presented data from the Stroop Task for which an analysis of mean RT misleadingly showed interference but no facilitation whereas a distribution analysis showed both interference and facilitation. Other studies have demonstrated that some models that adequately predict mean RT sometimes inadequately account for the shape of RT distributions (Hockley \& Corballis, 1982; Ratcliff \& Murdock, 1976). Also with distribution analyses, it was shown that some visual searches proceed both in serial and in parallel, whereas usual statistics yield to conclude that the search is sequential (Cousineau \& Shiffrin, 2003). Some authors have also demonstrated the need to take the shape of RT distributions into account when comparing the adequacy of different theoretical propositions (Hacker, 1980; Hockley, 1984; Ratcliff, 1978, 1979).

This article discusses how to characterize RT distributions in terms of probability density functions (especially using the ex-Gaussian) and how to implement the necessary analysis tools using MATLAB. Several key features make MATLAB a popular choice as a simulation package and analysis tool. First, the software runs on a variety of platforms, including Windows, Mac OS, LINUX and UNIX. Second, software implementations developed for one platform can easily be transposed to other platforms. Also, MATLAB provides a sophisticated computational environment with a simple, yet powerful, programming language, debugging tools, and a sophisticated graphical interface. Also, and not the least, MATLAB is computationally very efficient. Finally, a large community of scientists develops and shares tools for the MATLAB system.

The first part of this paper discusses the general problem of characterizing a frequency distribution of RT in terms of probabilistic functions. The general principles of maximum likelihood estimation for parameter estimation are then reviewed. A detailed mathematical tutorial on likelihood 
methods is presented in Myung (2003). In this paper, we present an implementation that allows fitting the popular ex-Gaussian function. The last section of the paper reports a Monte Carlo study that shows the validity of the proposed approach. Although the main focus of the present paper is the ex-Gaussian function, the general procedure described here can be used to estimate best fitting parameters of various probability functions. The proposed computational tools are written in MATLAB source code. They can be customized and run in any MATLAB environment and are available through the Internet at http://www. psy.ulaval.ca/ yves/distrib.html.

\section{Fitting Probability Density Functions to a Distribution of Response Times}

A probability density function (PDF) represents the distribution of values for a random variable. The area under the curve defined the density (or weight) of the function in a specific range. The density of a function is very similar to a probability. Characterizing a phenomenon in terms of PDF is very useful as it allows to estimate the probability that the process yields values in a specific range. Some phenomena in psychology are well described by PDF. For instance, the distribution of results for most standardized intelligence tests follows a Gaussian (normal) function. With a known mean and standard deviation, the probability that the results of an intelligence test are in a specific range of values can easily be computed. For example, a test characterized by a Gaussian distribution of results with a mean of 100 and a standard deviation of 15 implies that $50 \%$ of the population have results above the mean while an IQ above 145 (three standard deviations above the mean) corresponds to a very low probability.

The task of finding the parameter values of a probability function that best represents the distribution of empirical data is sometimes trivial. This is true, for example, when considering the Gaussian (normal) function. In this case, the

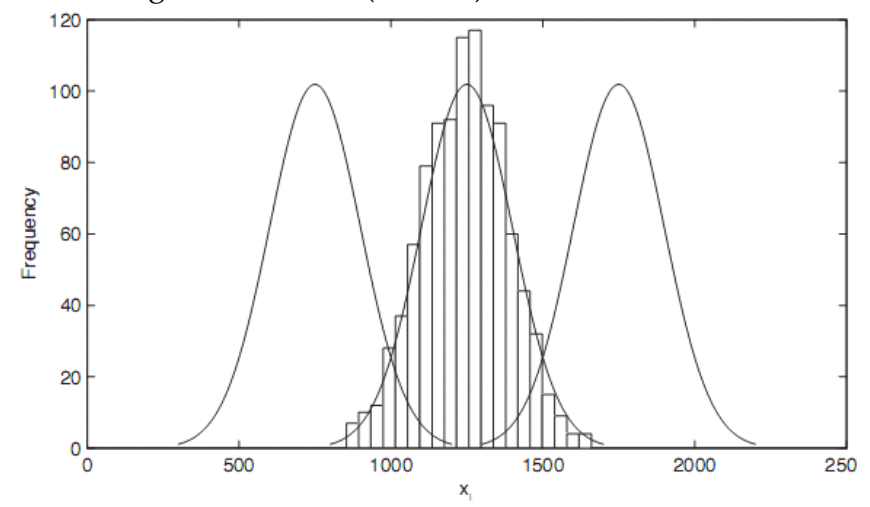

algebraic mean and standard deviation of the data are the most accurate estimates of the mean and standard deviation of the best-fitting Gaussian function. In other cases, the task of getting good estimations of the parameter values is more complex.

With non-trivial cases, like the popular ex-Gaussian function, an iterative approach allows the parameter space to be searched and the parameter values that best fit a frequency distribution to be estimated. To do this, the fit of the probability function to the data is evaluated using a goodness of fit (or error) criterion. An efficient and powerful fit criterion is the likelihood value (see Myung, 2003 for a tutorial). Given a data set and a PDF with specific parameter values, the likelihood criterion provides an indication of the fit between the data and the function. The best-fitting parameter values are associated with the greatest likelihood. This is why this statistical approach is known as maximum likelihood estimation. Given a PDF $\mathrm{f}(x \mid \theta)$ with $\mathrm{k}$ parameters $\theta=\left[\theta_{1}, \theta_{2}, \ldots, \theta_{k}\right]$ and an empirical set of data composed of $\mathrm{N}$ observations $\mathrm{x}_{\mathbf{i}}, \mathrm{i}=1, \ldots, \mathrm{N}$, the likelihood function is

$$
L(\theta \mid \mathrm{X})=\prod_{i=1}^{N} f\left(x_{i} \mid \theta\right),
$$

where $\Pi$ is the product operator. For large data sets, the computation of the likelihood value returns values close to zero and may provoke underflow errors. This is why the $\log$ of the likelihood criterion is often used instead of the likelihood criterion. The log transformation substitutes the sum operator to the product operator, less likely to provoke overflow errors. For historical reasons, minimization procedures were more widespread, so it became customary to minimize minus the log-likelihood instead of maximizing the log-likelihood. The minus log-likelihood criterion is

$$
\log L(\theta \mid \mathrm{X})=-\sum_{i=1}^{N} \ln \left[f\left(x_{i} \mid \theta\right)\right]
$$

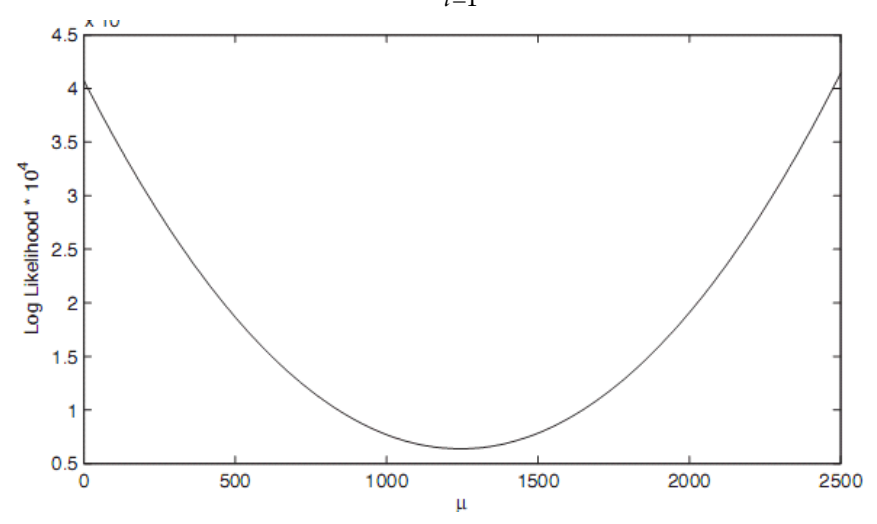

Figure 2. The minus log-likelihood criterion for the Gaussian distribution computed for various value of parameter $\mu$. The left panel shows the correspondence between the frequency distribution and the PDF functions with parameters $\mu_{\mathrm{A}}=750$, $\mu_{B}=1250$, and $\mu_{C}=1500$. The right panel shows how the LogL values change according to the value of parameter $\mu$. 
where $\ln$ is the natural logarithmic function. Figure 2 illustrates how the LogL criterion is minimized when the parameter values of a probability function best fit an empirical distribution. The histogram (top panel) represents a frequency distribution of $\mathrm{N}=100$ observations sampled from a random variable that follows a Gaussian PDF with a mean $\mu=1250$ and a standard deviation $\sigma=150$. The top panel also shows three overlay Gaussian PDFs (marked A, $B$, and C), which have different mean values of $\mu_{A}=750$, $\mu_{B}=1250$, and $\mu_{C}=1500$. The three Gaussian PDFs have the same standard deviation $\sigma=150$. Equation (2) allows $\log L$ to be computed according to the specific values of parameter $\mu$. For the Gaussian function,

$$
f\left(x_{i} \mid \mu, \sigma\right)=\frac{1}{\sigma \sqrt{2 \pi}} e^{-\frac{\left(x_{i}-\mu\right)^{2}}{2 \sigma^{2}}}
$$

and

$$
\log L(\mu, \sigma \mid X)=-\sum_{i=1}^{N} \ln \left[\frac{1}{\sigma \sqrt{2 \pi}} e^{-\frac{\left(x_{i}-\mu\right)^{2}}{2 \sigma^{2}}}\right]
$$

The computation yields $\log L\left(\mu_{\mathrm{A}} \mid \mathrm{X}\right)=11787$, $\log L(\mu \mathrm{B} \mid X)=6365$, and $\log L(\mu \mathrm{C} \mid X)=7835$. It can be seen that $\log L$ is smaller with parameter values that best fit the frequency distribution. The bottom panel of Figure 2 shows how, given the frequency distribution in the top panel, $\log L$ changes according to the value of parameter $\mu$. $\log L$ has a minimum value for $\mu=1250$, which corresponds to the bestfitting Gaussian function.

\section{Searching Parameter Spaces With the Simplex Method}

To find the parameter values that correspond to a minimum of the minus log-likelihood criterion, a search of the parameter space is required. Systematically trying all possible values would certainly be time consuming. This is especially true with functions that have several parameters defining a multi-dimensional space. Fortunately, there are general purpose algorithms such as the Simplex method that are robust and allow finding a minimum of a multiparameter function (see Press, Flannery, Teukolsky \& Vetterling, 1988, for a review of search methods and Cousineau, Brown and Heathcote, 2004 for a review in the context of distribution analyses). Using the $\log L$ criterion with the Simplex algorithm allows the best-fitting parameters of a PDF to a distribution of data to be found with great efficiency.

The LogL criterion defines a fit surface in a multiparameter space. Starting with predetermined parameter values, the Simplex method uses the steepest gradient on

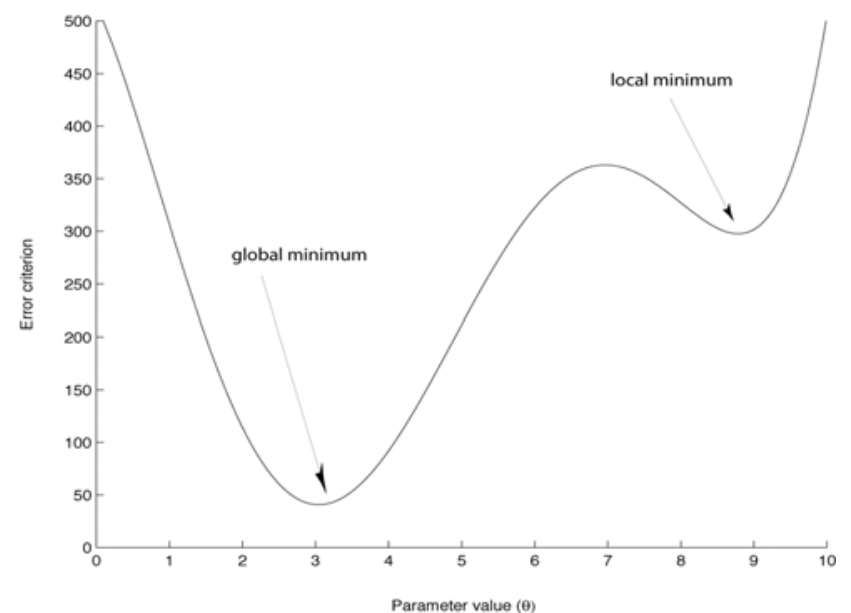

Figure 3. Global and local minima of a function: a search algorithm following the steepest gradient to find the minimum of $\mathrm{f}(x \mid \mu)$ might get stuck in the local minimum and converge without finding the global minimum.

the fit surface to determine how the parameter values should be changed to improve the fit of the adjusted function. The algorithm follows an iterative procedure that is applied until a minimum on the error surface is found. The steepest gradient corresponds to the steepest slope on the fit surface and, like a marble going down on a smooth surface, allows a minimum of the function to be found. The Simplex method works well providing that the fit surface is continuous and smooth. This is generally the case when using the LogL criterion with a reasonably large data set.

Typically, the search involves changes in parameter values that are made smaller at each iteration until the adjustment yields only small changes in the fit criterion. The search stops either when the improvement in fit is smaller then a pre-determined criterion or when the change in parameter values is smaller that another pre-determined value. The stopping criteria are called tolerances. The search is said to have reached convergence when the improvement in the goodness of fit is smaller than the termination tolerance or when the change in parameter values is smaller than the function tolerance.

\section{When the Search Fails to Converge: Local Minima and Erratic Error Surfaces}

Although the Simplex method is robust and efficient, the parameter search may fail to converge. This occurs when, after performing many iterations, the change in the fit criterion at a given iteration does not become smaller than the value of the termination tolerance or when the change in parameter values does not become smaller than the function tolerance. Two conditions may yield a failure of convergence. First, it is possible that the error surface is not smooth enough to allow a good adjustment of the parameter 
values. As mentioned before, this is usually not the case when using the LogL criterion for which the error surface is generally continuous and smooth. Second, it is possible that the search gets stuck in a local minimum. A local minimum is a region on the error surface that provides a minimum that is not a global minimum of the function. Figure 3 illustrates this situation. On this figure, the fit surface (vertical axis) is a function of a single parameter $\theta$ (horizontal axis). The function has a local minimum around parameter value $\theta=8.5$ and a global minimum for $\theta=3$. In this situation, like a marble rolling down the error surface, the search algorithm might get stuck in the local minimum and never reach the lower global minimum value. By doing a few big steps on the fit surface in the beginning of the search, most implementations of the Simplex method can usually detect a local minimum and find a global minimum. Nevertheless, a good strategy to avoid getting stuck in a local minimum is to start the search process with parameter values that are known to be in the neighborhood of the real parameter values. For example, with the popular exGaussian function, a reasonably good estimation of parameter $\mu$ is the difference between the algebraic mean and the skewness of the data. Using this value as a starting point for the search process reduces the risk of getting stuck in a local minimum. Even with reasonable starting values, the search may still fail to converge. When this happens, three things can be done to increase the probability of finding a global minimum of the function: (1) increase the maximum number of iterations allowed for the search process, (2) perform a new search with different starting parameter values, or (3) increase tolerances.

\section{A}

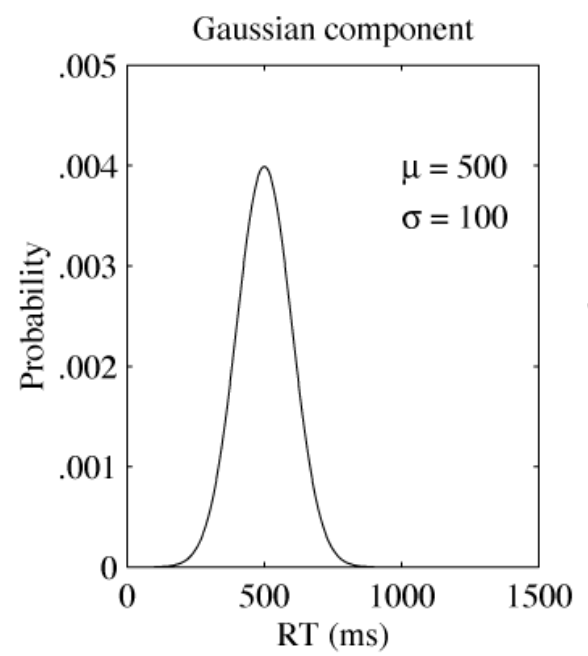

\section{Comparing the Fit of Different PDFs to the Same Distribution of Data}

The $\log L$ criterion does not allow the adequacies of different probability functions that have different number of parameters to be compared. For instance, the ex-Gaussian function has three parameters $(\mu, \sigma, \tau)$, the Gaussian two $(\mu, \sigma)$, and the Exponential function only one $(\tau)$. For a given data set, the best-fitting function is the one that yields the smallest $\log L$ value with the smallest number of parameters. Hélie (2006) discusses various fit criteria that allow comparing the fit of functions (or models) having different number of parameters. Among those fit criteria, Akaike's information criterion (AIC) is a popular goodness of fit indicator that takes into account the number of parameters. It is given by

$$
A I C=2 \log L(\theta \mid X)+2 k,
$$

where $\log L$ is the minus $\log$-likelihood value and $k$ is the number of parameters of the fitted function. AIC is smaller for probability functions with better fits. See Hélie (2006) for more details.

\section{The ex-Gaussian PDF}

The ex-Gaussian function is the convolution of two additive processes, a Gaussian (normal) function and an exponential function. Luce (1986, chap. 6) describes the exGaussian as a decision time model in cognitive processes. Mathematically, the ex-Gaussian probability density function is written as

$$
f(x \mid \mu, \sigma, \tau)=\frac{1}{\tau} \exp \left(\frac{\mu}{\tau}+\frac{\sigma^{2}}{2 \tau^{2}}-\frac{x}{\tau}\right) \Phi\left(\frac{x-\mu-\sigma^{2} / \tau}{\sigma}\right) .
$$

In this equation, the exponential function (exp) is multiplied by the value of the cumulative density of the Gaussian

B

\section{C}

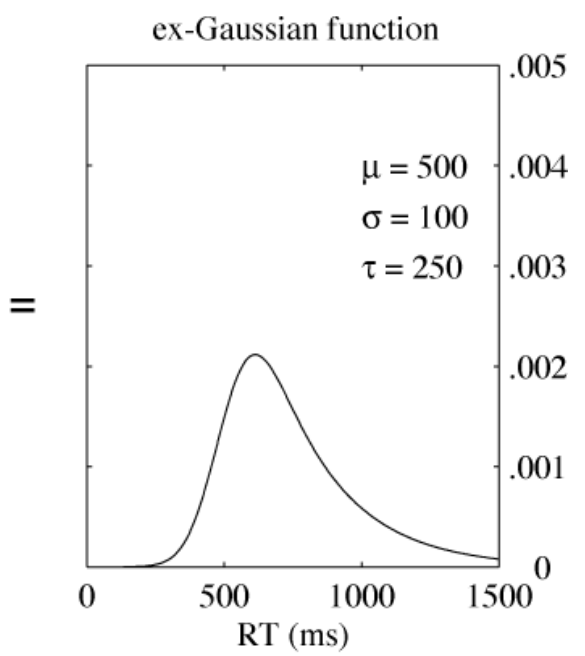

Figure 4. The ex-Gaussian probability function with parameters $\mu=500, \sigma=100$, and $\tau=250$ (Panel c) resulting from the convolution a Gaussian probability function (Panel A) with an exponential function (Panel B). 
function symbolized by $\Phi$. The resulting ex-Gaussian function has three parameters, $\mu, \sigma$, and $\tau$. The two first parameters ( $\mu$ and $\sigma$ ) correspond to the mean and standard deviation of the Gaussian component. The third parameter $(\tau)$ is the mean of the exponential component.

In the framework of cognitive processes, this convolution can be seen as representing the overall distribution of RT resulting from two additive or sequential processes. As proposed by Luce (1986, chap. 6), the exponential process can be seen as the decision component, i.e., the time required to decide which response to make, while the Gaussian component can be conceptualized as the transduction component, i.e., the sum of the time required by the sensory process and the time required to physically make the response. Although the theoretical proposition that RT of cognitive processes are the sum of two additive processes is difficult to test, several researchers have demonstrated that the ex-Gaussian function provides a very good fit to several empirical RT distributions (Ratcliff \& Murdock, 1976; Hockley, 1984; Luce, 1986). Other have emphasized that, in the absence of any theoretical assumptions, the ex-Gaussian function can effectively be used to characterize an arbitrary RT distribution (Heathcote et al., 1991). An interesting characteristic of the ex-Gaussian function is that its parameter values can easily be interpreted. Parameters $\mu$ and $\sigma$ are the mean and standard deviation of the Gaussian component and can readily be interpreted as localization and variability indicators. Parameter $\tau$ is the mean of the exponential component, which corresponds to the right 'tail' of the distribution; a larger $\tau$ implies a more skewed distribution.

Figure 4 illustrates how an ex-Gaussian function results from two additive processes. If the time (in $\mathrm{ms}$ ) required by the normal process has a mean $\mu=500$ and a standard deviation $\sigma=100$ (Panel A in Figure 4), and the time required for completion of the second process follows an exponential distribution with a mean $\tau=250$ (Panel B), then, for a given trial, the resulting RT is the sum of a value sampled from the Gaussian process and a value sampled from the exponential process. The resulting distribution of values follows the ex-Gaussian probability function with parameters $\mu=500, \sigma=100$, and $\tau=250$ (Panel C) and has a mean $\bar{x}=\mu+\tau$ and a standard deviation $s=\sqrt{\sigma^{2}+\tau^{2}}$.

Varying the parameters of the ex-Gaussian function leads to a continuum of shapes from 'pure' exponential to 'pure' Gaussian functions. Figure 5 presents three exGaussian curves with different parameter values. As

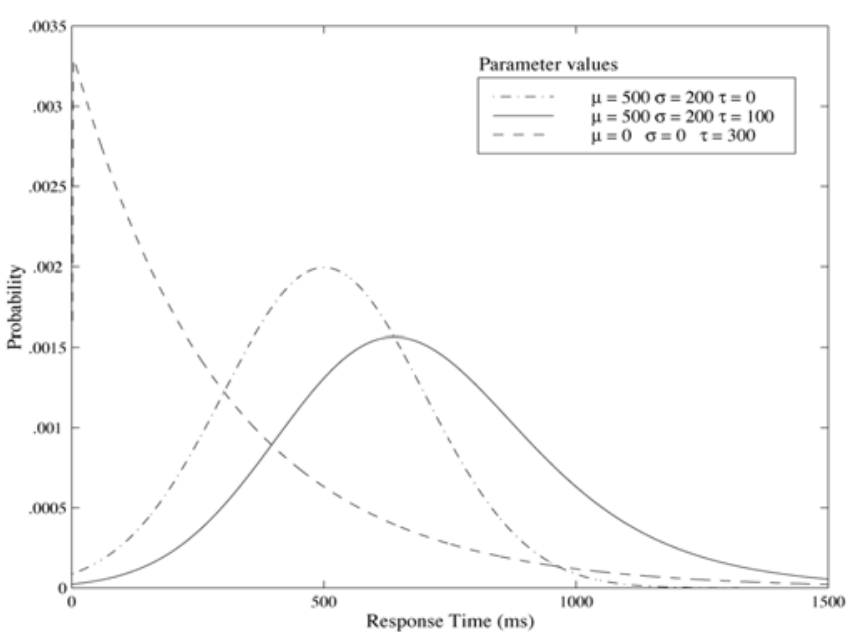

Figure 5. The ex-Gaussian PDF plotted for different parameter values.

illustrated, with parameters $\mu=0$ and $\sigma=0$, the ex-Gaussian function reduces to an exponential function. With parameter $\tau=0$, the ex-Gaussian function reduces to a normal function.

One difficulty with the ex-Gaussian function is that there is no arithmetic or other simple way to derive the parameters of the underlying processes from the observable data. To estimate the (unobservable) parameters, an iterative procedure like the one discussed above is used to find the parameter values for which the shape of the probability function best fits the frequency distribution of data.

\section{Using MATLAB to Fit the Ex-Gaussian Function to a Frequency Distribution of RT}

This section presents a MATLAB implementation that allows the popular ex-Gaussian function to be fitted to a frequency distribution of data. Implementing a search algorithm to fit the ex-Gaussian PDF to an empirical distribution requires three MATLAB functions:

1. A function implementing the ex-Gaussian PDF;

2. A function implementing the computation of the LogL criterion for the ex-Gaussian;

3. A search algorithm to find the best-fitting parameter values.

DISTRIB is a MATLAB toolbox comprising the necessary functions to fit the ex-Gaussian PDF. The complete set of functions is presented in Appendix 1, including exgausspdf.m, which implements the ex-Gaussian PDF. The function takes the following form: functionf=exgausspdf(mu,sigma,tau,data) 
Listing 1.the function simple_egfit

\title{
function R=simple_egfit(data)
}

\author{
ta $u=s t d(d a t a) * 0.8 ;$ \\ mu=mean(data)-skewness(data ); \\ sig =sqrt(var(data $)-(\operatorname{tau} \uparrow 2))$; \\ $\%$ reasonable starting value for tau \\ $\%$ reasonable starting value for mu \\ $\%$ reasonable starting value for sig \\ pinit $=[$ mu sig tau $]$; \\ $\%$ put starting parameter values in an array \\ $\%$ given the data, and pinit, find the parameter \\ $\%$ values that minimize eglike \\ $\%$ the function retums $\mathrm{R}=[\mathrm{mu}, \mathrm{sig}, \mathrm{ta} u]$ \\ $\mathrm{R}=\mathrm{fminse}$ rch(@(params) eglike(params,data),pinit);
}

Exgausspdf takes four arguments and returns the density of the ex-Gaussian PDFs with parameters mu, sigma, and tau, given the value in data. Arguments mu, sigma ( $>0)$, and tau $(>0)$ are scalars (numbers) and argument data is either a scalar, vector, or matrix. The function returns a vector of probabilities $f$ that has the same size as data.

Function eglike.m returns the likelihood value computed for the ex-Gaussian PDFs given specific parameter values and a data set. It has the following form: function $\log \mathrm{L}=$ eglike(params, data).

The function takes two arguments. Argument params $=[\mathrm{mu}$, sigma, tau $]$ is a three-element vector that specifies the parameter values of the ex-Gaussian function. The second argument is a vector of data for which the $\log \mathrm{L}$ value is computed. The function returns the minus log likelihood for the ex-Gaussian given the parameter values and data.

Function simple_egfit.m is a simple implementation that performs parameter searches using the Simplex method. This function is defined in Listing 1.

Simple_egfit takes a single input argument data (a

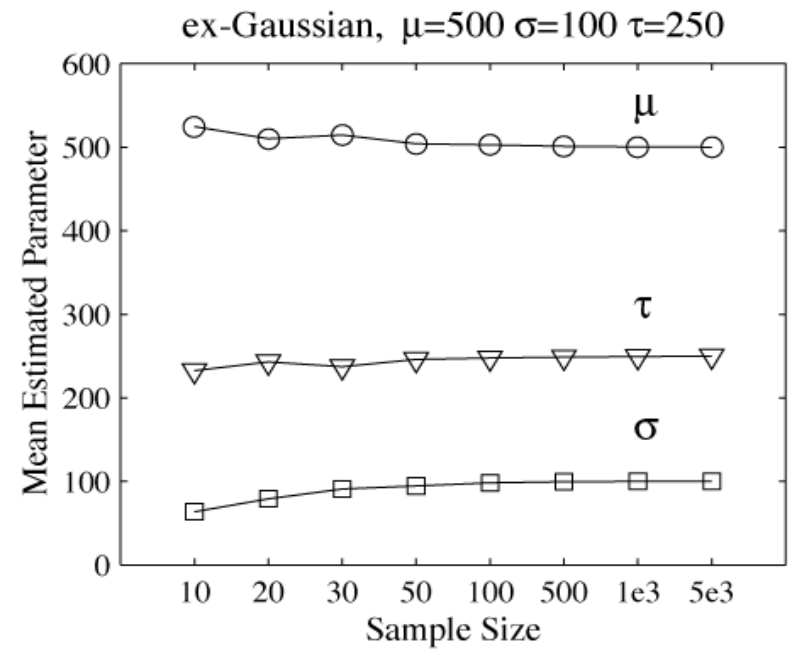

column vector of data) and returns a three-element vector $\mathrm{R}=[\mathrm{mu}$, sigma, tau $]$ made of the best-fitting ex-Gaussian parameter values. The starting values for the search procedure are estimated using simple heuristics. The search is done using MATLAB's fminsearch function that implements the Simplex search method. Function eglike is the fit criterion for the search process. Below is a description of fminsearch, MATLAB's implementation of the Simplex method.

\section{Fminsearch, a MATLAB Function that Implements the Simplex Method}

Fminsearch is a general purpose function that allows the minimum of a multi-parameter function to be found. To use fminsearch with the $\log L$ criterion, one needs to provide fminsearch with (1) the name of the function to be minimized, (2) starting parameter values for the search process, (3) the data to be fitted, and (4) some options that control the tolerances and the maximum number of iterations in the search process. If no options are provided, fminsearch works with default values. The format of

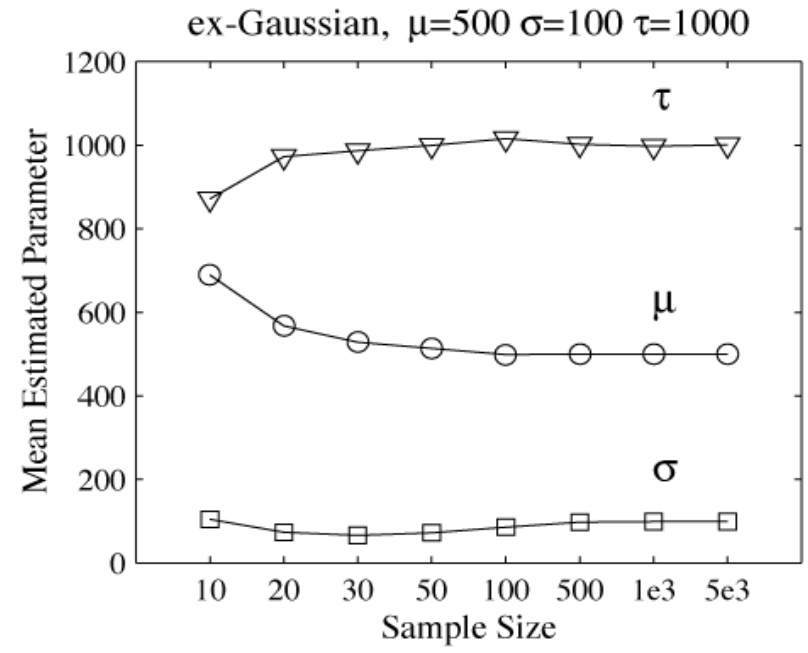

Figure 6 . Results of the Monte Carlo study: mean estimated parameter values. Results are reported according to sample size (10 to 5000) and are based on 1000 estimations. 
fminsearch is as follows:

$[\mathrm{x}, \mathrm{fval}$,exitflag, output $]=$ fminsearch(funfen, $\mathrm{x}$, options), where funfin is the function to be minimized, $x$ is a vector containing the starting parameter values for the search process, options is another vector containing some options that control the search process. Fminsearch returns a vector containing four elements [x,fval, exitflag, output]. Element $x$ contains the parameter values that correspond to a minimum of the objective function; element foal is the value of the minimized function given the data and returned parameters; exitflag and output provide additional information regarding the search process (for a detailed description see MATALB's Reference Guide).

In simple_egfit, fminsearch is called as follows:

$\mathrm{R}=$ fminsearch(@(params) eglike(params, data),pinit)

The string eglike(params, data) indicates that function eglike is to be minimized. Eglike return the likelihood for the ex-Gaussian function given the parameters in params $=[\mathrm{mu}$, sigma, tau $]$ and the actual data. The first part of the argument @(params) indicates which argument of eglike is to be estimated, in that case, the parameters in params while data is held constant. Also, pinit is a vector

Table 1. Results of the Monte Carlo study. Mean estimated parameters, standard errors, and confidence intervals for each theoretical distribution and sample size

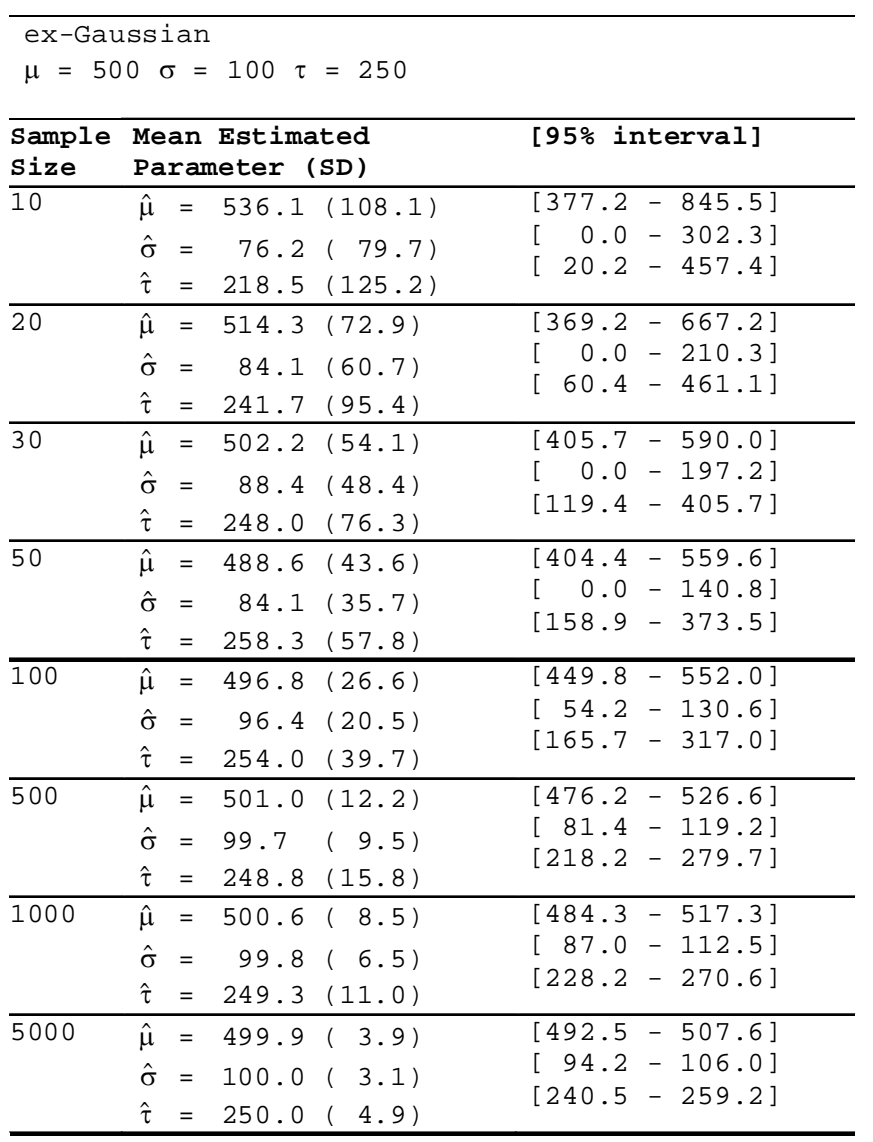

containing the starting parameter values used when the starting the search. In brief, the above syntax line calls function fminsearch to find the parameters value (params) for which function eglike show a minimum given the data. The search is done starting with the parameter values in pinit.

\section{Egfit.m, a More Robust Fitting Function for the Ex- Gaussian PDF}

Egfit is a MATLAB function that implements a more flexible and robust search process to fit the ex-Gaussian PDF to a frequency distribution. This function has the following form:

function $\mathrm{R}=$ egfit(data, params, options).

Egfit requires at least one argument data, which is a vector containing the data for which the ex-Gaussian is to be fitted. Two optional arguments, params and options, allow the search process to be controlled. Params $=[\mathrm{mu}$, sigma, tau] allows the starting parameter values for the search process to be specified, and options $=$ [ttolerance, ftolerance, niter] allows optional controls for fminsearch to be set. The optional tolerance values control the termination tolerance (ttolerance) and the functional tolerance (ftolerance) of the Simplex method. Default values are 0.0001. Lastly, optional argument niter controls the maximum number Table 1 (continued)

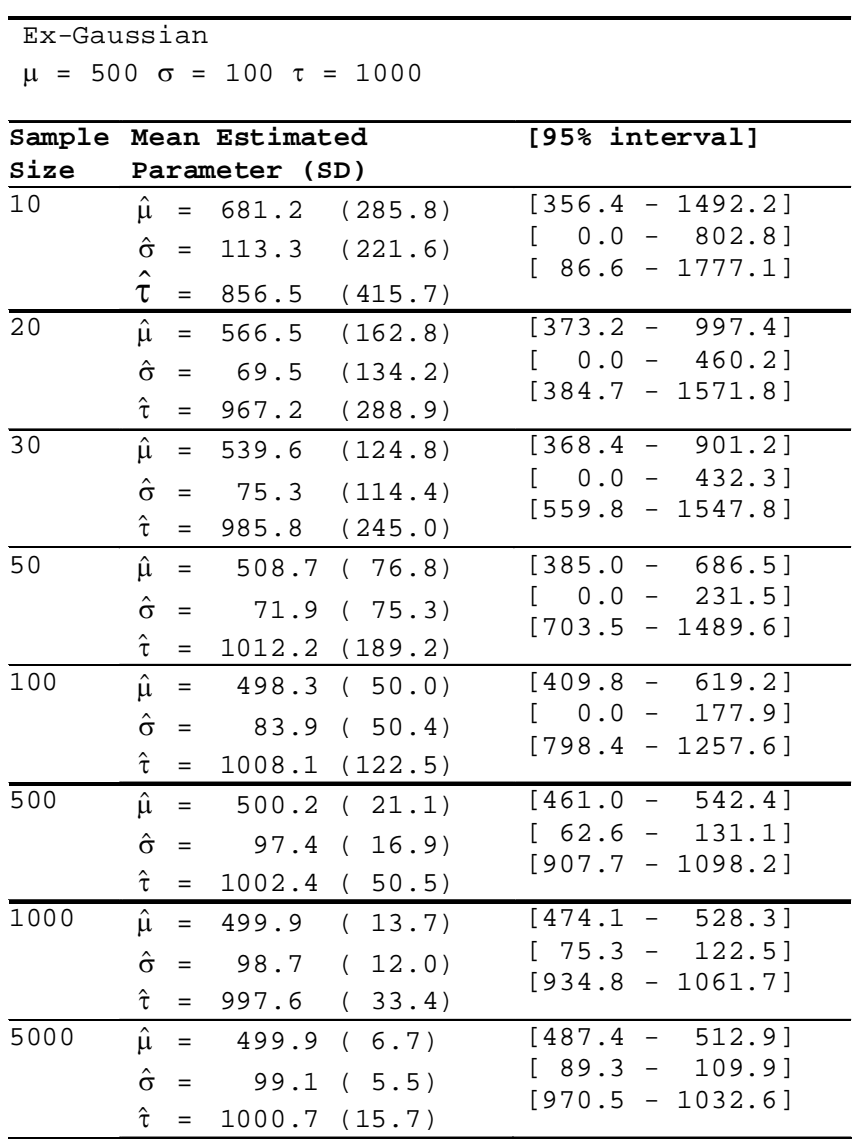


of iterations in the search process. The default value is niter $=200 *$ length(data).

\section{Testing egfit: a Monte Carlo Study}

A Monte Carlo study was performed to evaluate possible biases and estimate the standard error associated with the parameter estimates when using the present implementation of the search process. The general approach consisted of generating a large number of samples of fixed size by sampling an ex-Gaussian random variable with known parameter values. Parameter estimations were then obtained using egfit for each of the samples. This allowed a distribution of estimated parameter values to be reconstructed. For example, consider an ex-Gaussian function with some specific parameters. By generating 1000 samples of size 100 and estimating the parameter values for each sample, a good representation of the sampling distributions of the parameter values was obtained. The standard deviation of this sampling distribution corresponds to the standard error for a given parameter and sample size. Moreover, the mean of the sampling distribution can indicate possible biases. For an unbiased estimator, the mean of the sampling distribution should be close to the actual parameter value. A comprehensive and detailed Monte Carlo study of parameter estimation methods for popular probability functions is presented in Van Zandt (2000).

\section{Method}

The Monte Carlo study was performed using two sets of parameter values for the ex-Gaussian function (EG1 and EG2). For EG1, the parameter values were $\mu_{1}=500, \sigma_{1}=100$, and $\tau_{1}=250$, and for EG2, they were $\mu_{2}=500, \sigma_{2}=100$, and $\tau_{2}=1000$.

For each of the two theoretical distributions, the Monte

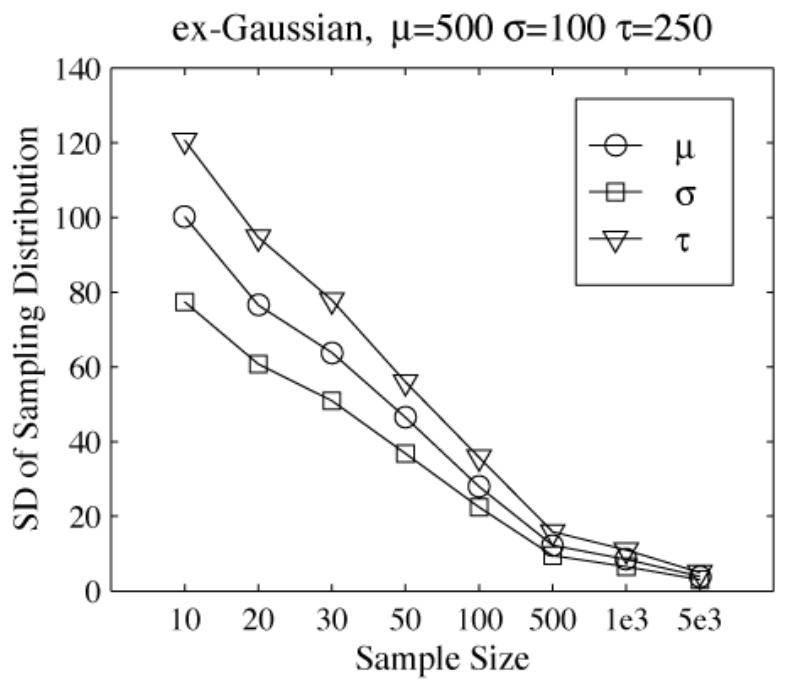

Carlo study was performed for sample sizes $\mathrm{N}=10,20,30$, 50, 100, 500, 1000, and 5000. A Monte Carlo estimation of the sampling distribution was obtained for each sample size by sampling the theoretical distribution one thousand times and performing a likelihood estimation of the parameters for each sample. Sixteen Monte Carlo simulations (two functions, EG1 and EG2 × 8 sample sizes), each based on 1000 samples, were performed.

\section{Results}

For each of the two theoretical functions, the sampling distribution of each parameter was constructed for each sample size. Table 1 summarizes the results. For each sample size, the mean and standard deviation of the sampling distribution are presented along with a $95 \%$ confidence interval based on the observed percentile values of the distribution. Figure 6 shows the average estimated parameter values plotted according to sample size for each distribution.

The sampling distributions show some small biases for smaller sample sizes. For the specific parameter values used in this Monte Carlo study, the means of the sampling distributions do not appear strongly biased for sample sizes of 100 or more. Figure 7 presents the standard deviations of the sampling distributions (the standard error) plotted according to sample size. For both of the ex-Gaussian distributions, the results show a monotonic decrease with an increase in sample size. The decrease in standard deviation with increasing sample size is sharp up to sample size $\mathrm{N}=$ 500. The Monte Carlo study shows that egfit provides good parameter estimations for the ex-Gaussian function, at least for the test values that were used.

\section{Conclusion}

This paper discusses the problem of characterizing RT

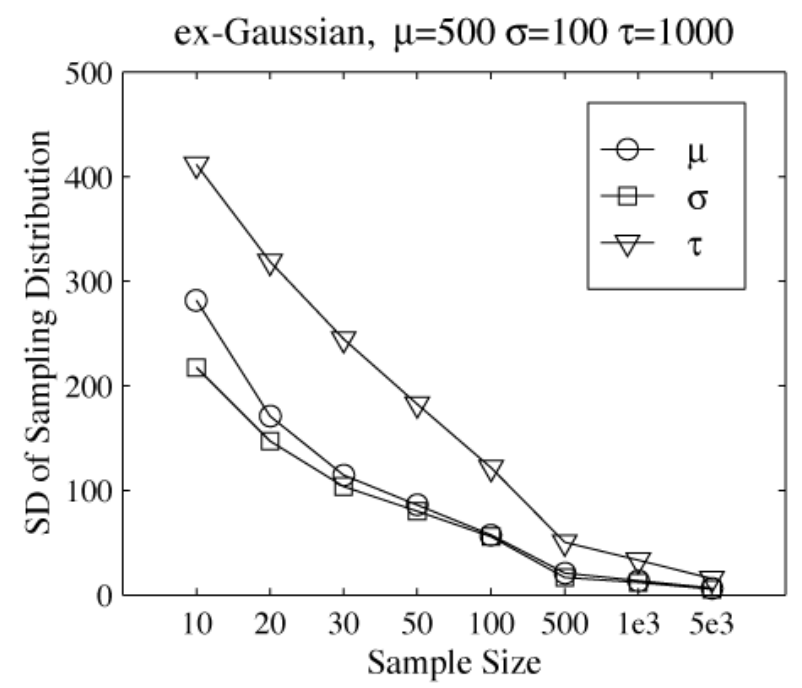

Figure 7. Results of the Monte Carlo study: standard deviation of the estimated sampling distributions. Results are reported for each parameter according to sample size (10 to 5000) and are based on 1000 estimations. 
distributions in terms of probability functions. The functions implemented in this paper provide simple and efficient ways to estimate parameter values of the ex-Gaussian function. A Monte-Carlo study has shown the validity of the proposed implementation. Other implementations can be developed with the same approach using the MATLAB fminsearch function, as discussed in the present paper.

\section{References}

Cousineau, D., Brown, S., \& Heathcote, A. (2004). Fitting distributions using maximum likelihood: Methods and packages. Behavior Research Methods, Instruments, $\mathcal{E}$ Computers, 36, 742-756.

Cousineau, D., \& Shiffrin, R. M. (2004). Termination of a visual search with large display size effect. Spatial Vision, 17, 327-352.

Hacker, M. J. (1980). Speed and accuracy of recency judgments for events in short-term memory. Journal of Experimental Psychology: Human Learning and Memory, 6, 651-675.

Heathcote, A., Popiel, A. J., \& Mewhort, D. J. K. (1991). Analysis of response time distributions: An example using the Stroop Task. Psychological Bulletin, 109, 340-347.

Hélie, S. (2006). An introduction to model selections: Tools and algorithms. Tutorials in Quantitative Methods for Psychology, 2, 1-10.

Hockley, W. E. (1984). Analysis of response time distributions in the study of cognitive processes. Journal of Experimental Psychology: Learning, Memory and Cognition, 6, 598-615.
Hockley, W. E. \& Corballis, M. C. (1982). Test of serial scanning in item recognition. Canadian Journal of Psychology, 36, 189-212.

Luce, R. D. (1986). Response times. New York: Oxford University Press.

Myung, I.J. (2003). Tutorial on maximum likelihood estimation. Journal of Mathematical Psychology. Vol 47(1), 90-100.

Press, W. H., Flannery, B. P., Teukolsky, S. A. \& Vetterling, W. T. (1988). Numerical recipes: The art of scientific computing. Cambridge, England: Cambridge University Press.

Ratcliff, R. (1978). A theory of memory retrieval. Psychological Review, 85, 59-108.

Ratcliff, R. (1979). Group reaction time distributions and an analysis of distribution statistics. Psychological Bulletin, 86, 446-461.

Ratcliff, R. \& Murdock, B. B. (1976). Retrieval processes in recognition memory. Psychological Review, 83, 190-214.

Van Zandt, T. (2000). How to fit a reaction time distribution. Psychonomic Bulletin and Review, 7(3), 424-465.

Manuscript received February 23, 2007

Appendix follows 


\section{Appendix}

Description of the functions in DISTRIB, a MATLAB toolbox implementing the fitting process for the ex-Gaussian PDF

\begin{tabular}{|c|c|}
\hline a ic.m & $\begin{array}{l}y=a \text { ic }(\log l, k) \\
\text { Given the minus log likelihood value } \log l \text { and the number of parameters } k \text {, returns } \\
\text { Akaine's information criterion. Both arguments must be the same size. }\end{array}$ \\
\hline simple_egfit.m & $\begin{array}{l}\mathrm{R}=\text { simple_egfit(data) } \\
\text { Simple implementation of the search process to fit the ex-Gaussian function using } \\
\text { MATLAB's implementation of the Simplex search method. Argument data is a column } \\
\text { vector containing the data to be fitted. The function returns a maximum likelihood } \\
\text { estimate of parameter values for the data in hand. } \\
\mathrm{R}=[\mathrm{mu}, \text { sig, tau }] \text { contains the three estimated ex-Gaussian parameters. }\end{array}$ \\
\hline egfit.m & $\begin{array}{l}\mathrm{R}=\text { egfit(data) } \\
\mathrm{R}=\text { egfit(data, params, options) } \\
\mathrm{R}=\text { egfit(data, [mu, sig, tau], [ttolerance, ftolerance, niter]) } \\
\text { Does a parameter search for the ex-Gaussian function using MATLAB's implementation of } \\
\text { the Simplex search method. This implementation is more robust and flexible than function } \\
\text { simple_egfit. Argument data is a column or row vector containing the data to be fitted. } \\
\text { Optional argument params }=[\mathrm{mu}, \text { sig, tau] allows starting parameter values for the search } \\
\text { process to be specified; optional argument options = [ttolerance, ftolerance, niter] allows } \\
\text { optional controls for fminsearch to be set; The optional tolerances control the termination } \\
\text { tolerance (ttolerance) and the functional tolerance (ftolerance). Default tolerance is } 0.0001 . \\
\text { Optional argument niter controls the maximum number of iterations in the search process. } \\
\text { The default value is niter }=200 * \text { length(data). } \\
R=[m u, \text { sig, tau] contains the three estimated ex-Gaussian parameters. }\end{array}$ \\
\hline eglike.m & $\begin{array}{l}\text { logl = eglike (params, data }) \\
\text { Given parameters params }=[m u, \text { sigma, tau }] \text { and vector data, returns the minus log- } \\
\text { likelihood value for the ex-Gaussian function. }\end{array}$ \\
\hline exga usspdf.m & $\begin{array}{l}\mathrm{f}=\operatorname{exg} \text { a usspdf(mu, sig, ta } \mathrm{u}, \mathrm{x}) \\
\text { Returns the probability at value } x \text { of the density function for the ex-Gaussian distribution } \\
\text { with parameters } m u \text {, sig, and tau. }\end{array}$ \\
\hline exppdf.m & $\begin{array}{l}y=\operatorname{exppdf}(x, m u) \\
\text { Returns the probability at value } x \text { of the density function for the exponential distribution } \\
\text { with parameter } m u \text {. }\end{array}$ \\
\hline plotegfit.m & $\begin{array}{l}\text { plotegfit(params, data) } \\
\text { Plots an histogram of data vector with overlay ex-Gaussian PDFs with parameters } \\
\text { params }=[m u \text {, sigma, tau }] \text {. }\end{array}$ \\
\hline & $p=p n f(x, m u$, sig $)$ \\
\hline
\end{tabular}

Returns the cumulative value, observed at $x$, for the density function of the Gaussian distribution with parameters $m u$ and sig. 